\title{
EVALUACIÓN DE MURO VERDE EN LA DISMINUCIÓN DE RUIDO Y SU PERCEPCIÓN AMBIENTAL EN UN CENTRO EDUCATIVO
}

\author{
EVALUATION OF GREEN WALL IN THE DECREASE OF NOISE AND ITS \\ ENVIRONMENTAL PERCEPTION IN AN EDUCATIONAL CENTER
}

\begin{abstract}
Ana Claudia Zeballos Pimentel ${ }^{1}$
Carmen Rosa Román Arce ${ }^{2}$
\end{abstract}

Información del artículo:

Recibido: 12/02/2020

Aceptado: 20/06/2020

\footnotetext{
${ }^{1}$ Ingeniero Ambiental. Universidad Privada de Tacna. E-mail: zeballosanaclaudia@gmail.com

${ }^{2}$ Escuela de ingeniería Ambiental, Universidad Privada de Tacna. E-mail: carromana@upt.pe
} 


\section{Resumen}

El estudio tuvo como objetivo instalar un muro verde con la finalidad de realizar un análisis comparativo de los niveles de disminución de ruido, así como evaluar la percepción ambiental El estudio fue realizado en la ciudad de Tacna en las instalaciones de institución educativa Verdad y Vida - Veritas et Vita. Para la construcción del muro verde se empleó la Aptenia cordifolia que fue instalada en los exteriores del centro educativo, para evaluar reducción de ruido se ubicó dos puntos de monitoreo; uno en dirección de la fuente de ruido y el otro detrás del muro verde hacia donde se dirige el ruido.

Se realizaron monitoreo en horarios nocturnos y diurnos, resultando con una reducción promedio en el horario diurno de 2,593 $\pm 0,47$ y en horario nocturno de 5,38 $\pm 0,19$ en ambos casos significativo ( $p$-valor $<0,05$ ). La percepción ambiental evaluada por la población estudiantil indica que el $50 \%$ percibe que los muros verdes reducen el ruido. Se concluye que los muros verdes pueden reducir el ruido y que podrían servir de pantalla acústica, siendo la Aptenia cordifolia, una buena alterativa.

Palabras claves: Calidad ambiental; Muro verde; Percepción ambiental; Ruido.

\section{Abstract}

The study aimed to install a green wall in order to carry out a comparative analysis of the levels of noise reduction, as well as to evaluate the environmental perception; the study was carried out in the city of Tacna in the facilities of the educational institution Verdad y Vida Veritas et Vita. For the construction of the green wall, the Aptenia cordifolia that was installed outside the educational center was used. In order to noise reduction monitoring, two monitoring points were located; one in the direction of the noise source and the other behind the green wall where the noise is directed.

Monitoring was carried out at night and daytime, resulting in a reduction in daytime of $2,593 \pm 0.47$ and at night of $5.38 \pm 0.19$ in both cases significant ( $p$-value -0.05 ). The environmental perception evaluated by the student population indicates that $50 \%$ perceive that green walls reduce noise. It is concluded that green walls can reduce noise and that they could serve as an acoustic screen, with Aptenia cordifolia being a good alternative.

Keywords: Environmental Quality; Green wall; Environmental perception, Noise. 


\section{Introducción}

En la actualidad la gran mayoría de personas viven en ciudades, esto conlleva a una disminución a la Calidad Ambiental por la gran demanda de recursos. Ello es motivo de buscar alternativas para poder crear ciudades sostenibles. En las ciudades por su dinamismo existe alto tránsito de personas y especialmente tráfico vehicular que causan problemas de excesivos ruidos que inciden en la calidad de vida de los habitantes. En la búsqueda alternativas que reduzcan los ruidos ambientales se han investigado la forma de reducirlo en la fuente o disminuir sus efectos con la instalación de pantallas acústicas (Pfretzschner, J., Simón, F., Moreno, A., de la Colina, C., y Rodríguez, R., 1996; Teira Arnoso, A., 2013; Alba, J., Del Rey, R., Torres, J. V., Bertó, L., y Hervás, C., 2012 y Romero Ibáñez, A., 2011). Entre los materiales utilizados clasifican a la utilización de vegetación por su interesante capacidad que tiene para absorberla, además de su función estética, entre sus cualidades en conjunto destaca sus propiedades aislantes gracias a la combinación de la capa de tierra, actualmente existen diferentes modelos que facilitan su uso y se utilizan en fachadas de edificios, casas, colegios, etc.

Así mismo destaca en su fabricación los muros verdes que proveen espacios verdes y mejor calidad ambiental en las ciudades, así en diferentes estudios (Posada, Arroyave \& Fernández (2009); López (2016) Fernández (2008),; Cáceres \& Vareta (2018)), concluyen que son alternativas viables que pueden disminuir ruidos que afectan a la población

En la ciudad de Tacna, con el paso de los años el parque automotor incrementó sostenidamente, siendo una preocupación latente por sus efectos negativos como la generación de ruido, especialmente en zonas que necesitan estar exenta de ruidos, como caso particular las zonas donde se sitúan las instituciones educativas.

\section{Objetivos}

El objetivo de la investigación evaluar un muro verde a fabricada a partir de Aptenia cordifolia, con la finalidad de disminuir el ruido exterior que afecta a la institución educativa Verdad y Vida, situada en una avenida de alto tránsito, así como pósito evaluar la percepción que tienen los estudiantes del desempeño del muro verde en la reducción del ruido.

\section{Metodología}

Para el monitoreo de ruido y medir niveles de presión sonora, se utilizó el sonómetro normado utilizado para medir niveles de presión sonora. MINAM (2013), este es un instrumento, siendo la unidad de medida el decibelio. Así mismo para poder determinar la percepción ambiental, se aplicaron encuestas a los alumnos de la institución educativa Particular Verdad y Vida ubicada en el cercado de Tacna, donde fue instalado el muro verde.

Para el muro verde se utilizó la especie fue la Aptenia cordifolia es un tipo de planta rastrera comúnmente utilizada en sistemas de cobertura para protección de laderas, se caracteriza por tener un rápido crecimiento y bajo requerimiento hídrico. Los tallos pueden llegar a medir hasta 60 centímetros postrados o colgantes, al ser una planta suculenta sus hojas están engrosadas porque almacenan agua y tienen una forma plana y peciolada de 1 a 3 centímetros con forma de corazón.

Se construyeron dos estructuras metálicas de 136 por 175 y 105 por $175 \mathrm{~cm}$ y de espesor $5 \mathrm{~cm}$ en ambos casos, así mismo se puso una funda de malla Raschel 65 \% Litec SKU: 25501 que es una red tejida de polietileno de alta densidad con 40 bolsillos de $12 \times 18 \mathrm{~cm}$ para poder colocar la planta. En estas estructuras se denominadas "Muros Verde", se instaló riego tecnificado usando mangueras a 16 
$\mathrm{mm}$ en cada bolsillo alimentado mediante un tanque de agua, dicho sistema se puede observar en la figura 1. Una vez instalado el muro verde, es necesario el mantenimiento adecuado, con el fin que el muro esté en óptimas condiciones, la poda del follaje cada 3 meses, para extraer las hojas dañadas y no dejar crecer tanto los tallos.

\section{Figura 1}

Muro verde construido a partir de Aptenia cordifolia

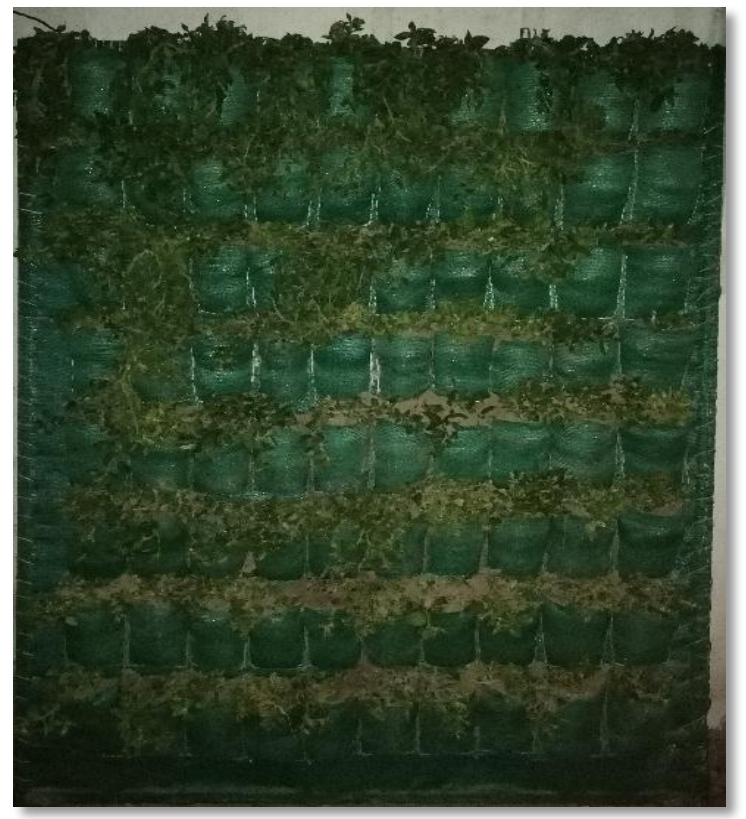

Nota. Diagramación propia.

Para determinar la cantidad de aislamiento, se empleó la diferencia entre los niveles sonoros que se obtienen con muro verde y sin muro verde, a esto se le conoce como aislamiento acústico del área.

\section{Cantidad de Aislamiento}

$$
\text { Qais }=P 1-P 2
$$

Donde:

Qais = Cantidad de aislamiento

$\mathrm{P} 1=$ Nivel sonoro 1

P2 = Nivel sonoro 2 


\section{Resultados}

Las mediciones del ruido se realizaron en tres días diferentes y en dos horarios; el diurno y nocturno con 10 repeticiones en cada uno, se contrastó en dos puntos el ruido exterior (punto 1) con el ruido interior o detrás del muro verde (punto 2), los datos se muestran en la tabla 1.

\section{Tabla 1}

Medición de ruido en tres días por horarios y puntos (dBA equ.)

\begin{tabular}{|c|c|c|c|c|c|c|c|c|c|c|c|c|}
\hline \multirow{3}{*}{$\begin{array}{c}\text { Tiempo } \\
\text { (Repeticiones) }\end{array}$} & \multicolumn{4}{|c|}{ Día 1} & \multicolumn{4}{|c|}{ Día 2} & \multicolumn{4}{|c|}{ Dia3 } \\
\hline & \multicolumn{2}{|c|}{ Diurno } & \multicolumn{2}{|c|}{ Nocturno } & \multicolumn{2}{|c|}{ Diurno } & \multicolumn{2}{|c|}{ Nocturno } & \multicolumn{2}{|c|}{ Diurno } & \multicolumn{2}{|c|}{ Nocturno } \\
\hline & Punto 1 & Punto 2 & Punto 1 & Punto 2 & Punto 1 & Punto 2 & Punto 1 & Punto 2 & Punto 1 & Punto 2 & Punto 1 & Punto 2 \\
\hline 1 & 64,1 & 63,6 & 58,5 & 68,4 & 66,9 & 72,5 & 51,1 & 62,2 & 68,3 & 68,6 & 59,5 & 68,3 \\
\hline 2 & 64,4 & 68,5 & 62,6 & 66,4 & 67,3 & 67,0 & 64,6 & 69,2 & 67,1 & 73,4 & 62,6 & 66,4 \\
\hline 3 & 64,1 & 70,9 & 67,2 & 68,9 & 66,7 & 70,8 & 52,7 & 61,4 & 67,5 & 69,0 & 67,2 & 68,9 \\
\hline 4 & 67,9 & 68,3 & 61,6 & 67,5 & 68,1 & 69,9 & 64,2 & 54,9 & 69,1 & 69,0 & 61,7 & 67,4 \\
\hline 5 & 66,4 & 72,1 & 59,1 & 68,3 & 66,1 & 69,7 & 59,5 & 65,3 & 68,6 & 70,0 & 59,1 & 68,3 \\
\hline 6 & 63,4 & 66,8 & 59,5 & 67,3 & 68,1 & 71,0 & 61,2 & 65,7 & 67,1 & 72,3 & 59,6 & 67,3 \\
\hline 7 & 64,0 & 69,2 & 65,6 & 57,4 & 67,2 & 59,3 & 58,2 & 62,4 & 67,7 & 69,6 & 65,6 & 57,2 \\
\hline 8 & 65,4 & 68,5 & 62,7 & 69,8 & 68,0 & 70,3 & 58,8 & 68,0 & 65,3 & 69,1 & 61,7 & 69,8 \\
\hline 9 & 65,2 & 69,9 & 59,8 & 68,7 & 66,0 & 69,7 & 59,2 & 67,5 & 66,6 & 70,3 & 59,8 & 68,7 \\
\hline 10 & 64,8 & 65,7 & 62,6 & 68,7 & 66,1 & 67,7 & 62,2 & 69,5 & 67,1 & 69,7 & 62,7 & 68,8 \\
\hline Promedio & 65,0 & 68,4 & 61,9 & 67,1 & 67,1 & 68,8 & 59,2 & 64,6 & 67,4 & 70,1 & 62,0 & 67,1 \\
\hline
\end{tabular}

Nota. Los estándares de calidad ambiental (ECA) fijan como valores máximos 40 dB para nocturno y 50 dB diurno. Las mediciones fueron efectuadas los días 08/11/2019, 11/11/2019 y 12/11/2019, El punto 1 de la medición se efectuó directamente a la fuente de emisión y el punto 2 detrás del muro verde.

En la figura 1 se observa que los monitoreo de ruido realizados en los tres dias y turno en general muestran una reduccion, ello se evidencia en las diferencias que existen entre los puntos 1 y 2 , no obstate no disminuyen hasta valores inferiores a lo indicado en los ECA's, si se observa una aparente disminucion en casi todos los dias.

Es importante determinar la atenuación acústica originada por el muro verde, con ese objetivo se realizó una comparación estadística que se observa en la tabla 2, en general en los tres días de medición se observa la reducción de ruido de forma significativa, excepto el día dos y horario diurno (pvalue<0,05). 


\section{Figura 2}

Diferencias en la reduccion de ruido según dias de medicíon y estandares de calidad (ECA)
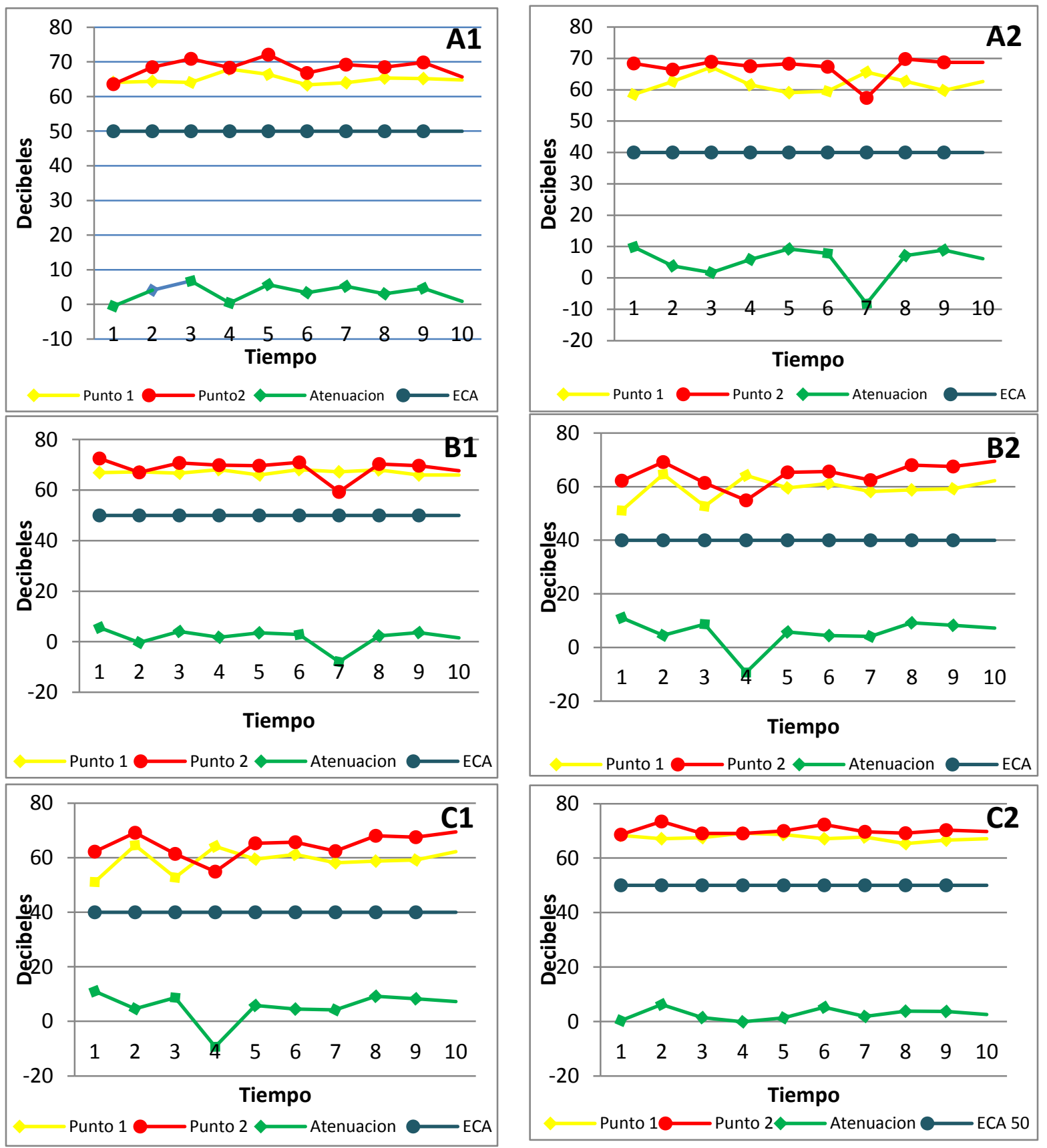

Nota. $40 \mathrm{~dB}$ para nocturno y 50 para diurno. A1 y A2 mediciones realizadas el primer dia, B1 y B2 medicones realizadas el segundo dia y $\mathrm{C} 1$ y $\mathrm{C} 2$ mediciones realziadas el tercer dia. 


\section{Tabla 2}

Resumen estadístico de atenuación de ruido por días

\begin{tabular}{llccc}
\hline Día & $\begin{array}{c}\text { Desviación } \\
\text { estándar }\end{array}$ & $\begin{array}{c}\text { Atenuación } \\
\text { acústica }\end{array}$ & -value \\
\hline 1 & Diurno & 2,42 & $3,38 \pm 0,77$ & 0,0014 \\
& Nocturno & 5,35 & $5,22 \pm 1,70$ & 0,0019 \\
2 & Diurno & 3,75 & $1,74 \pm 1,20$ & 0,1626 \\
& Nocturno & 5,67 & $5,44 \pm 1,80$ & 0,0110 \\
3 & Diurno & 2,08 & $2,66 \pm 0,66$ & 0,0003 \\
& Nocturno & 5,35 & $5,16 \pm 1,70$ & 0,0020 \\
\hline
\end{tabular}

Nota. Obtenido de reporte de mediciones.

Respecto la percepción ambiental determinada mediante encuestas a estudiantes de la institución educativa donde se instaló el muro verde, afirmaron les preocupa mucho el medio ambiente en un $90 \%$, mientras que el $80 \%$ conoce de los benéficos de muros verdes, no obstante el $50 \%$ percibe que no atenúa el ruido.

\section{Discusión}

Los muros verdes se han identificado como aquellos que sirven para atenuar el ruido en fachadas, también tienen fines estéticos tal como afirman Rivas-Sánchez, Pérez, y Roldán-Cañas (2018) y Rivas-Sánchez (2020). La especie Aptenia Cordifolia, tiene dichas propiedades que pueden ayudar a mejorar la calidad ambiental en las urbes, por tanto a la luz de los resultados se puede afirmar en el estudio se determinó que la especie atenúa el ruido, resultados similares han sido obtenido por Delgadillo (2018) y Ccepaya (2018) quienes emplearon la misma especie para atenuar el ruido en condiciones controladas, no obstante la reducción no es menor o por debajo de lo normado en las ECA's, existe una disminución significativa al $95 \%$ de confianza

\section{Conclusión}

Los resultados expuestos en la investigación permitirían concluir que la especie Aptenia Cordifolia, no solo demuestran la efectividad como muros verdes para el aumento de áreas verdes si no que pueden ayudan a mitigar los efectos adversos de los ruidos existentes, determinándose una reducción promedio en el horario diurno de 2,593 \pm ,47 y en horario nocturno de 5,38 $\pm 0,19$ en ambos casos significativo ( $p$-valor $<0,05$ ). Por lo tanto se recomienda el uso de la especie para ser usado en la construcción de muros verdes. 


\section{Referencias Bibliográficas}

Alba, J., Del Rey, R., Torres, J. V., Bertó, L., \& Hervás, C. (2012). Cámara de transmisión acústica a escala para el estudio de pantallas acústicas. In Proceedings of the VIII Congreso Iberoamericano de Acústica, el VII Congreso Ibérico de Acústica, el 430 Congreso Español AcústicaTecniacústica (pp. 1-11).

Ccepaya Loayza, Y. Y. (2018). Eficiencia de las plantas ornamentales Aptenia Cordifolia y Helxine Soleirolii como barrera para la reducción de ruido 2018.

Delgadillo Valdez, G. J. (2018). Reducción del ruido mediante barreras vegetales con las especies Jacobaea maritima y Aptenia cordifolia en condiciones controladas-Lima 2018.

Fernández, M. Y. (2008). Una revisión de la literatura mexicana con énfasis en Áreas Naturales Protegidas. Espiral, Estudios sobre Estado y Sociedad

López, B. T. (2016). Jardines Verticales. Valencia: Escuela Técnica Superior de Arquitectura.

Marchesi E. (2000). Plantas Ornamentales. Uruguay: Nuestra Tierra.

Mendoza, C. G. (2017). Mejoramiento del confort climático de una vivienda mediante techos ecológicos con Aptenia Cordifolia, San Juan de Lurigancho-2017. Lima: Universidad César Vallejo.

Posada, I., Arroyave, M. d., \& Fernández, C. (2009). Influencia de la Vegetación en los niveles de ruido urbano. Colombia: Revista EIA, ISSN 1794-1237.

Pfretzschner, J., Simón, F., Moreno, A., de la Colina, C., \& Rodríguez, R. (1996). Pantallas acústicas absorbentes realizadas con granzas de goma. Tecniacústica, 96, 251-254.

Rivas-Sánchez, Y. A. (2020). Eficiencia del uso de muros verdes para disminuir los efectos negativos de la pérdida de áreas verdes en las ciudades con el uso de materiales reciclados.

Rivas-Sánchez, Y. A., Pérez, M. F. M., \& Roldán-Cañas, J. (2018) Uso De Sustratos Alternativos Para Mejorar La Retención Y Distribución De Agua En Muros Verdes.

Romero Ibáñez, A. (2011). Estudio de la reducción del ruido aerodinámico de trenes de alta velocidad con pantallas acústicas. Universidad de Cantabria.

Teira Arnoso, A. (2013). Adecuación y validación de una cámara reverberante a escala para ensayos de pantallas acústicas. 